\title{
Biological and pretreatment processes for the valorization of waste and biomass into energy/fuel and useful materials - WasteEng2008, 3-5 June 2008, Patras (Greece)
}

The WasteEng Conference Series (http://www.wasteeng.org/) promotes the sustainable valorization of waste and biomass for the production of energy and useful materials, with emphasis on processes and practices that reduce emissions and life cycle assessment of technologies and end-of-pipe products. The very successful 2008 issue (WasteEng08, Patras, Greece) has shown the richness of this ever growing field. This special issue presents a selection of contributions dealing with the utilization of primarily biological and pretreatment processes for the production of energy/fuels and useful materials from waste and biomass. For this purpose, this issue covers a wide variety of cases (waste and biomass streams), processes and approaches.

The main bulk of the presented work deals with the use of anaerobic biological processes for the exploitation of agroindustrial waste and biomass. Anaerobic digestion is a classical process for the production of biogas from waste and biomass. The works of Carrere et al. and Batimelli et al. present novel methods of improving biogas yields from pig manure and slaughterhouse waste, respectively. The work of Kabouris et al. examines the possibility to codigest municipal sludge with FOG (fat, oil and grease) waste for the production of biogas. Lovanh et al. present information on the performance of an anaerobic lagoon treating swine wastes.

An alternative to direct anaerobic digestion is a two-stage process that produces biohydrogen in the first reactor and biogas in a second reactor, which is a normal anaerobic digester. The works of Venetsaneas et al. and Koutrouli et al. present such two-stage processes for the production of gaseous biofuels from cheese whey and olive pulp, respectively. Alternately, a two-stages process producing biohydrogen (first stage) and biopolymers (second stage) from olive-mill wastewater is possible (Ntaikou et al.). Zhang et al. present a mechanochemical method for the production of hydrogen from cellulose. Koutinas et al. demonstrate how cheese whey may be utilized to produce starter cultures for the dairy industry, while Romdhana et al. present a fry-drying method for the energetic valorization of industrial biomass.

Composting represents a significant option for the valorization of waste and biomass. Chroni et al. investigate the microbial community structure and activity as indicators for the composting process, while Tremier et al. examine the effect of the initial physical characteristics of the material to be composted (sludge) on the composting performance.

Goncalves et al. examine the ability of nonconventional yeasts to detoxify olive-mill wastewaters, a first important step towards valorization of this waste. Sarasa et al. examine the biodegradability of a bioplastic material waste, while Prado et al. present their findings in the toxicity and biodegradation of tetracycline in a membrane bioreactor.

Biodiesel is of course one of the main alternative fuels produced these days. Glycerine is an important by-product of biodiesel production. The valorization of this main by-product is certainly crucial for rendering biodiesel production economically viable. The work of Galan et al. presents a method for valorizing glycerin for the production of triacetin.

In summary, this special issue gathers papers addressing the valorization of various wastes and biomass using various technologies. This makes this issue of the WasteEng08 Conference on biological and pretreatment processes for the valorization of waste and biomass into energy and useful materials a very interesting contribution.

Prof. Gerasimos Lyberatos University of Patras,

Greece

Prof. Ange Nzihou Ecole des Mines d'Albi-Carmaux, France 